\title{
Probing Galaxy Growth and Dusty Star-Forming Systems at $z>1.5$ across Diverse Environments in the Stripe82/HETDEX Field with Herschel
}

\author{
Rebecca Larson ${ }^{* a}$, Shardha Jogee ${ }^{a}$, Nicholas Watson ${ }^{a}$, Marco Viero ${ }^{b}$, Tim Weinzirl ${ }^{c}$, \\ Harold W. Yorke ${ }^{d}$, Steve Finkelstein ${ }^{a}$, Casey Papovich ${ }^{e}$, Caitlin M. Casey ${ }^{a, f}$, Robin \\ Ciardullo $^{g}$, Caryl Gronwall ${ }^{g}$, Stephanie LaMassa ${ }^{h}$, and C. Meg Urry ${ }^{h}$ \\ ${ }^{a}$ Astronomy, University of Texas at Austin, Austin, TX, United States. \\ ${ }^{b}$ Stanford University, Stanford, CA, United States. \\ ${ }^{c}$ University of Nottingham, University Park, Nottingham, United Kingdom. \\ ${ }^{d}$ NASA Jet Propulsion Lab (JPL), Pasadena, CA, United States. \\ e Texas A\&M University, College Station, TX, United States. \\ ${ }^{f}$ University of California, Irvine, Irvine, CA, United States. \\ $g$ Pennsylvania State University, State College, PA, United States. \\ ${ }^{h}$ Yale University, New Haven, CT, United States. \\ E-mail: saturnswingsegmail.com, sjeastro.as.utexas.edu
}

\begin{abstract}
In the next few years, we will embark on an unprecedented study of how a million galaxies grow their stars and dark matter halos over a large comoving volume $\left(0.5 \mathrm{Gpc}^{3}\right)$ in the cosmic web at the epoch $(1.9<z<3.5)$ where cosmic star formation and black hole activity peak, and proto-clusters start to collapse. This study is enabled by the powerful synergy of six photometric and spectroscopic surveys, which are providing Herschel SPIRE, Spitzer IRAC, NEWFIRM Kband, DECam ugriz, and XMM X-ray imaging data, along with future optical spectroscopic data from HETDEX over a very large area $\left(28 \mathrm{deg}^{2}\right)$ in the Stripe82/HETDEX field. In this work, we illustrate the power of these combined datasets and focus on studying dusty, star-forming systems (DSFSs) identified by the Herschel Stripe 82 Survey (HerS). Using the 250, 350, and 500 micron SPIRE data over our $28 \mathrm{deg}^{2}$ field, we identify a number of possible high redshift $(z>4)$ DSFSs which will be prime candidates for follow-up observations. We discuss their properties and possible association with galaxies and quasars detected at X-ray, IR, optical, and UV wavelengths. We discuss broader implications for galaxy growth at early cosmic times.
\end{abstract}

Frank N. Bash Symposium 2015

18-20 October

The University of Texas at Austin, USA

* Speaker. 
We are currently conducting an unprecedented study of how a large sample of $\sim 1$ million galaxies grow at redshifts of $1.9<\mathrm{z}<3.5$ across a wide range of environments, from low-density fields to massive proto-clusters, within a huge co-moving volume of $0.5 \mathrm{Gpc}^{3}$ in the SHELA/HETDEX $28 \mathrm{deg}^{2}$ legacy field. This study is enabled by the synergy of our six deep wide-area photometric surveys (DECam/ugriz, Spitzer/IRAC, NEWFIRM/K, Herschel/SPIRE, Chandra, and XMM), with the upcoming Hobby-Eberly Telescope Dark Energy Experiment (HETDEX) blind spectroscopic survey.The dataset will yield photometric redshifts, stellar mass, star formation rates, black hole accretion rates, spectroscopic redshifts, galaxy clustering, biases, and dark halo masses. This paper focuses on our ongoing analysis of the Herschel/SPIRE Stripe 82 Survey (HerS) data which traces obscured dusty star-forming systems. Below are some highlights from our study focusing on HerS.

- The HerS Herschel SPIRE data detects 13,324 sources at 250 microns in our $28 \mathrm{deg}^{2}$ field. At $\mathrm{z} \sim 2$, the data can detect infrared-luminous galaxies (ULIRGs and hyper-LIRGs) with SFRs above several hundred $\mathrm{M}_{\odot} \mathrm{yr}^{-1}$. By applying the Dowell et al. (2013) method to the Herschel data, we identify 91 candidate galaxies at $\mathrm{z}>4$ in the $28 \mathrm{deg}^{2}$ field. For an assumed redshift range of $4<z<6$, the corresponding number density of dusty galaxies is around $10^{-7}$ galaxies $\mathrm{Mpc}^{-3}$. This low value is between that of sub-millimeter galaxies and high-z radio galaxies at $\mathrm{z} \sim 2-4$. We plan future follow-up observations at other wavelengths to confirm the redshift and explore the nature of these $\mathrm{z}>4$ systems.

- Our Herschel/SPIRE data from the HerS survey (Viero et al. 2014) are crucial for studying dusty star- forming systems, many of which are not detected in the UV or optical data. In such systems, dust blocks UV and optical light, and heats up to re-emit far infrared radiation. The Spitzer/IRAC data at 3.6 and 4.5 microns are sensitive to existing stars and constrain the stellar mass. For these galaxies the far-infrared photometry is key as there is a lack of corresponding UV emission.

- A candidate high-redshift $(\mathrm{z}>2$ ) dusty star-forming system has been identified in the Herschel 250, 350, and $500 \mu \mathrm{m}$ data with corresponding the beam FWHMs of 18”, 25", and 36 ". The 250 micron 18" beam contains many (sometimes $>3$ ) separate sources identified in the Spitzer/IRAC 3.6 micron and DECam/ugriz data whose resolution is much higher (2"). Note that the difference in resolution poses a major challenge and that a single far-IR source is often associated with many IR and optical sources. The problem in resolution can lead to severe inaccuracies in estimating galaxy number densities from sub-millimeter surveys.

- We are currently characterizing the star formation rates and other properties of the dusty star-forming systems via fits to their spectral energy distributions (SEDs), which has been completed for one of the galaxies in this survey identified to be at $\mathrm{z}>2$. This galaxy 's SED estimates a total IR luminosity (LIR) of $2 \times 10^{13} \mathrm{~L}_{\odot}$, and a dust temperature $\left(\mathrm{T}_{d}\right)$ of $20.9 \mathrm{~K}$. These values are consistent with a galaxy at a redshift $\mathrm{z} \sim 2.5$. This particular galaxy's SED estimates a star formation rate (SFR) of $1650 \mathrm{M}_{\odot} \mathrm{yr}^{-1}$ and a stellar mass $\left(\mathrm{M}_{*}\right)$ of $10^{12} \mathrm{M}_{\odot}$. These values are consistent with hyper-LIRGs and is an example of one of the most extreme star-forming objects in the universe.

$R L$ and SJ acknowledge support from the National Aeronautics and Space Administration (NASA) JPL SURP Program and NSF grant NSF AST-1413652. 


\section{References}

[1] C.D. Dowell et al, HerMES: Candidate High-redshift Galaxies Discovered with Herschel/SPIRE, ApJ 780, 75 (2013) [arxiv. org/abs/1310.7583].

[2] M. Viero et al, The Herschel Stripe 82 Survey (HerS): Maps and Early Catalog, ApJs 210, 22 (2014) [arxiv.org/abs/1308.4399]. 